\title{
Recruitment of a Large Community of Pediatricians in a Collaborative Research Project
}

\author{
Lawrence F. Nazarian, MD, ${ }^{*}$ Lois A. Maiman, PhD, ${ }^{*}$ Marshall H. Becker, PhD, MPH†
}

\begin{abstract}
To investigate the effects of an educational program on the compliance-enhancing behavior of pediatricians and the subsequent regimen adherence of their patients, it was necessary to enlist the participation of a large number of pediatric practitioners. The physicians had to be motivated to attend two evenings of tutorial training; to enroll their patients in the project; and to complete detailed study documents. Ultimately, 90 of the 97 pediatricians practicing in the community took part.

The following factors are felt to have contributed to the widespread participation of the pediatricians: 1) the subject was interesting and of relevance to clinical practice; 2) the study was designed so as not to interfere with office routine; 3) major demands were not made on the patients; 4) due consideration was given to the value of the physicians' participation; and 5) communication between the investigators and the practitioners was facilitated by a liaison pediatrician.
\end{abstract}

\section{$T$}

HE MEDICAL LITERATURE contains a substantial body of research resulting from the efforts of practicing physicians. ${ }^{1-4}$ This type of investigation has been conducted for years in the Rochester, New York pediatric community, often involving close collaboration among academicians and practitioners. ${ }^{5}$ The study described in this article is unique in that it involved the participation of nearly all of the 97 practicing pediatricians in the Greater Rochester Area. Lessons gained from the design and conduct of this project should be of value in the planning and execution of future collaborative studies.

From the *Department of Pediatrics, University of Rochester School of Medicine and Dentistry, Rochester, New York; and the $\dagger$ Department of Health Behavior and Health Education, University of Michigan School of Public Health, Ann Arbor, Michigan.

Correspondence to: Lawrence F. Nazarian, MD, Department of Pediatrics, Box 777, University of Rochester, School of Medicine and Dentistry, 601 Elmwood Avenue, Rochester, NY 14642.

Supported by grants from the National Center for Health Services Research and Health Care Technology Assessment, Department of Health and Human Services (HS0 4897) and the National Institute of Child Health and Human Development, National Institutes of Health (HD00538) (Dr. Maiman).

Received for publication September 1988, revised October 1988 , and accepted January 1989.

\section{Study Description}

This research addressed the question of whether or not educating the pediatrician in techniques for improving patient cooperation would lead to measurable improvements in pediatricians' compliance-enhancing behaviors, and to increased regimen adherence by mothers. Although the study methods and results are described elsewhere, ${ }^{6}$ a brief description is presented to put physician recruitment into perspective.

Participating pediatricians were randomly assigned to either a control group or one of two Continuing Medical Education (CME) interventions. One-third attended two evening tutorials on strategies for improving patient compliance, taught by an individual with extensive knowledge in this area. Specially-designed printed materials summarizing the tutorial content supplemented these teaching sessions. Another one-third received only the printed materials. The remaining participants served as controls, receiving neither instruction nor materials.

After the educational phase of the study was completed, the pediatricians or their office staff recruited 
mothers of children presenting with acute otitis media. They told the mothers that they were participating in a study of the treatment of ear infections and asked them to agree to a subsequent home interview. That interview was conducted approximately 1 week after the office visit by a research assistant. It focused on techniques the pediatrician might have employed during the office visit to influence adherence, and assessments of mothers' compliance were obtained.

In the final study phase, the pediatricians filled out a questionnaire and answered a quiz pertaining to their knowledge, attitudes, and practices regarding compliance. They also supplied information from their medical records as to whether follow-up otitis appointments were kept by the children in the study.

\section{Methods of Pediatrician Recruitment}

The study began with the identification of all practicing pediatricians in the Rochester area. A pediatrician was included if at least 50 percent of his or her professional time was spent in general pediatric practice.

A liaison physician was selected to communicate directly with the physicians and represent the principal investigators. This individual was a pediatrician who, at the time of the study, had practiced in the area for 15 years, and who knew most of the community pediatricians personally. It was agreed that blocks of time would be allotted for the specific purpose of pediatrician recruitment and that these efforts would be reimbursed by the project.

During a 1 -month period, the liaison physician contacted all of the identified pediatricians, either by telephone or in person. The proposed study was discussed briefly, with careful description of what would be expected of the participants, and with emphasis on the potential benefits of study results to pediatric practice. The pediatricians were promised that follow-up data on the study's overall findings, and on their own individual practices, would be supplied to all participants when the study was completed. It was made clear that agreement to participate might involve attendance at tutorials, and that all physicians would need to recruit patients and complete questionnaires. At that time, tentative indication of interest in the study was solicited.

Shortly after this initial contact, a follow-up letter was sent, requesting formal commitment by way of a return post card. If the card was not returned, a second mailing was sent. If there was still no response, a telephone call was made by the liaison physician.

Research involving the general pediatric commuyears in the Rochesunique in that it inctitioners in the

Participating pediatricians were randomly assigned to study groups, and individuals assigned to the tutorial group were offered a choice of three different session times so as to increase convenience (and thus likelihood) of attendance.

For the patient recruitment phase, a goal of eight patients per pediatrician was set. This figure was carefully selected as a balance of research needs and realistic demands on the pediatricians and practices. Physicians enrolling patients at a very slow rate were called by the liaison physician and encouraged to intensify their efforts.

In the final study phase, self-administered questionnaires and inquiries about follow-up appointment-keeping were sent to the pediatricians. A second mailing was made to nonresponders, followed by a telephone call from the liaison physician to those not answering the mailings.

The liaison physician devoted a total of 48 hours to his responsibilities over the course of the study. Time was expended on conferences with the other investigators, several long sessions involving large numbers of telephone calls, and many sporadic contacts with participants as the need arose. He was compensated at $\$ 50$ per hour, for a total of $\$ 2,400$. The budget for the entire study was $\$ 230,000$.

\section{Results of Recruitment Efforts}

\section{Enrollment}

At the time of the study, there were 97 pediatricians practicing in the Greater Rochester Area (which includes the city and surrounding suburbs in a 30-mile radius) who met the inclusion criteria. All but four expressed interest at the time of initial contact. One cited scheduling difficulties as a reason for nonparticipation, and three had concerns about the study itself.

The first enrollment letter yielded 82 responses and the second brought seven more. The remaining 
eight physicians received follow-up telephone calls and seven of these returned the post card. A total of 91 pediatricians formally agreed to participate; one was later dropped because geographical distance of the office would make patient home visits difficult.

The six nonparticipants were interviewed after the study was completed. They offered the following reasons for not enrolling: too busy; would not participate in projects not citing his name as author; felt the study itself was not worthwhile (two partners); would not be available during the tutorial schedule; and had recently undergone heart surgery.

\section{Tutorial Attendance}

Thirty-seven pediatricians were randomly assigned to the tutorial group. Of these, four had scheduling conflicts and were reassigned to the mailed printed materials group. All of the remaining 33 physicians attended both sessions of the tutorial - a total of 5 hours-with the exception of one who missed one session because of illness.

\section{Patient Recruitment}

Although there was a goal of eight patients for each participating pediatrician, a physician was kept in the study analysis if data were available on at least four patients. Eighty-three participants met this criterion, while seven recruited three or fewer. Interviews conducted at the completion of the study revealed the following reasons for the low numbers of patients recruited by those seven physicians: often forgot about the study while busy with practice concerns; did not want to take the extra time; recruitment interfered with daily routine; topic under study was not compelling; and relatively small number of patients falling into the study category. These findings suggest the value of efforts to continually monitor patient recruitment and to assist the participating physician in this task.

\section{Follow-up Documents}

Questionnaires containing 62 items were sent to the 90 participants. The initial mailing yielded 58 responses, and a second mailing, supplemented by telephone calls, brought in 30 more, for a total of 88 completed questionnaires.

Each physician was asked to supply data on patient follow-up otitis appointment keeping. Since one physician had recruited no patients, 89 tally sheets were sent out. After two mailings followed by telephone calls, all 89 were completed and returned.

A supportive relationship between "town" and "gown" can facilitate collaborative clinical research and teaching.

\section{Discussion}

This research project provides an example of how widespread initial and continued participation in a clinical study can be achieved within a medical community. Several factors contributed to the investigators' success in obtaining the cooperation of so many busy physicians.

The subject of the study was of interest to practitioners. If a physician is persuaded that what is being studied is relevant to everyday practice, he or she is much more likely to respond with enthusiasm. Patient adherence is of concern to practitioners, and the added dimension of trying to improve the situation by educating the physician stimulated further interest. Most physicians enjoy CME, and the opportunity to attend a tutorial by an authority in the field was appealing. Also, participants knew that they would ultimately receive feedback from the research, both in terms of general findings and data specific to their own practices.

The study did not make major demands on the physician. All practitioners are busy, and therefore reluctant to further burden their schedules. By keeping the physician's role modest, the investigators made participation less onerous. There is a natural tendency to include in research designs additional elements to examine still more aspects of the problem being studied. (A study done in our department on bacteremia in febrile children was elegantly and painstakingly set up, but was so comprehensive and detailed that a total of one patient was ultimately enrolled!)In the present instance, the actual work required to generate necessary data was anticipated and requested, and the participants knew what to expect. Even so, the phase that was most demanding-patient recruitment-involved simultaneous patient care and research effort.

The study did not make major demands on the patients. Patients often enjoy participation in research, but will be reluctant if their own routines are disrupted or if they see the project as threatening or painful. (An example is a study on meningococcal vaccine 
that was timed to begin just after the publicity emerged on the side-effects of swine flu vaccine; recruitment of patients was extremely difficult.) By conducting the interview in the patient's own home, the investigators made participation convenient for the patient and also made it easier for physicians to enlist patient cooperation.

There was consideration of the value of the physician's participation. The study design was constructed with obvious recognition of busy schedules. Feedback to the participants was incorporated into the study plan. Although the physicians recognized the value of the study and the opportunity to learn and required no further recompense, the investigators' awareness of their needs and circumstances was appreciated.

A liaison physician facilitated communication between the investigators and the practitioners. This individual knows most of the pediatricians personally and, as a busy practicing physician, had the additional advantage of credibility. He helped the investigators construct a study plan that would minimize infringement on the participants' daily activities. Past experience has shown that studies conducted in offices require, in the planning stages, the viewpoint of the practitioner who must integrate the research with usual patient care. It was easier for the liaison physician to encourage and gently prod the physicians than it would have been for the academic researchers themselves. Because these activities can require considerable time to be performed correctly-similar to the effort devoted by investigators to development of methods and data analysis-it was important that compensation be budgeted for such effort.

There is an ongoing relationship between the sponsoring academic institution and the community physicians. All practicing pediatricians have a clinical faculty appointment at the University of Rochester School of Medicine and Dentistry, and do considerable clinical teaching both in their offices and at the university-affiliated hospitals. Research in practice, often in collaboration with full-time faculty, has been conducted in the Rochester community for decades and has been facilitated by a supportive relationship between "town" and "gown" physicians. This kind of interaction exists in some medical communities but is not present in many. The experience described in this paper provides one example of why such a relationship should be fostered.

As a final thought, it is evident that many of the factors that elicit physician participation in a study are the same factors that enhance patient adherence in a therapeutic program. A one-to-one approach by a trusted individual, personal conviction that the treatment (or topic) is important, consideration of the patient's (or study participant's) needs with minimal infringement on lifestyle, and gentle encouragement and reminders are all elements that contribute to the successful outcome of both clinical therapies and collaborative studies.

\section{Acknowledgment}

The authors would like to thank the pediatricians of the Greater Rochester area for their outstanding support of this project.

\section{References}

1. Pickles WN. Research in general practice. Br Med J 1948;2:469-71.

2. Style AJ. Research in the family physician's office. J Natl Med Assoc 1977;69:783-5.

3. Breese BB. Research in the practice of a pediatrician. Am J Dis Child 1961;101:413-22.

4. Nazarian, LF. Research in pediatric practice. Ped Clin Nor Am 1981;28:585-99.

5. Hoekelman RA, Klein SW, McInerny TK, et al. Collaborative research between a department of pediatrics and its clinical faculty. Clin Pediatr 1979;18:623-8.

6. Maiman LA, Becker MH, Liptak GS, et al. Improving pediatricians' compliance-enhancing practices: a randomized trial. Am J Dis Child 1988;142:773-9. 